Research Article

\title{
An Uncertain Alternating Renewal Insurance Risk Model
}

\author{
Jia Zhai $\mathbb{D}^{1},{ }^{1}$ Haitao Zheng $\mathbb{D}^{2},{ }^{2}$ Manying Bai $\mathbb{D}^{,},{ }^{2}$ and Yunyun Jiang ${ }^{3}$ \\ ${ }^{1}$ School of Economics, Beijing International Studies University, Beijing 100024, China \\ ${ }^{2}$ School of Economics and Management, Beihang University, Beijing 100191, China \\ ${ }^{3}$ School of Economics, Peking University, Beijing 100871, China \\ Correspondence should be addressed to Haitao Zheng; zhenghaitao@buaa.edu.cn
}

Received 17 April 2020; Accepted 20 May 2020; Published 6 July 2020

Guest Editor: Wenguang Yu

Copyright ( $) 2020$ Jia Zhai et al. This is an open access article distributed under the Creative Commons Attribution License, which permits unrestricted use, distribution, and reproduction in any medium, provided the original work is properly cited.

\begin{abstract}
The claim process in an insurance risk model with uncertainty is traditionally described by an uncertain renewal reward process. However, the claim process actually includes two processes, which are called the report process and the payment process, respectively. An alternative way is to describe the claim process by an uncertain alternating renewal reward process. Therefore, this paper proposes an insurance risk model under uncertain measure in which the claim process is supposed to be an alternating renewal reward process and the premium process is regarded as a renewal reward process. Then, the paper also gives the inverse uncertainty distribution of the insurance risk process. The expression of ruin index and the uncertainty distribution of the ruin time are derived which both have explicit expressions based on given uncertainty distributions. Finally, several examples are provided to illustrate the modeling ideas.
\end{abstract}

\section{Introduction}

The classical insurance risk models and their extended models generally assume that claim numbers and claim amounts are random variables. They also suppose that the time of the accident and the time of payment are consistent; that is, the insurance company immediately pays compensation to the insured when the accident occurs. Then, many types of insurance risk models are presented by means of stochastic process based on the probability theory; several scholars, for example, Dickson and Hipp [1], Li and Garrido [2], Dickson and Hipp [3], Chun [4], Gerber and Shiu [5], Sundt and Teugels [6], Paulsen and Gjessing [7], Albrecher and Hipp [8], and Yu et al. [9], extend the insurance risk model by considering inflation, dividend, and tax. Using Lévy process to model insurance risk processes and other insurance product has become popular, see, for example, Griffin [10], Biffis and Kyprianou [11], Zhang et al. [12], and $\mathrm{Yu}$ et al. [13].

However, for a new insurance product, it usually lacks historical data to estimate probability distributions. In this situation, we often use the belief degrees of the claim numbers and claim amounts estimated by some experienced domain experts to describe the indeterminacy. Kahneman and Tversky [14] find that humans tend to place too much emphasis on unlikely events. Thus, probability theory is difficult to model the belief degree unless we get enough historical data, and it is unreasonable to describe an insurance risk process with stochastic process. The research about the insurance risk process that is described by a stochastic process has been gradually challenged by many scholars. De Wit [15] first developed an insurance risk process under fuzzy theory. Then, the insurance risk processes with fuzziness are studied by Lemaire [16], Cummins and Derrig [17], Derrig and Ostaszewski [18], Yu [19], Shapiro [20], and Li et al. [21]. Furthermore, Huang et al. [22] and Shapiro [23] regard the claim amounts as fuzzy random variable and propose the fuzzy random risk model.

When the estimated distributions are not close enough to the real frequencies, Liu [24] invented the uncertainty theory, which is used to model human indeterminacy due to belief degrees. Now, the theory has been applied to construct insurance risk models. Considering the human uncertainty in running an insurance company, Li et al. [25] propose a premium principle under uncertain measure via the distortion function. To study the evolution of uncertain 
phenomenon over time, Liu [26] develops the concept of uncertain process. Meanwhile, Liu also presents the uncertain renewal process as a special and important case. After that, Liu [27] researches the uncertain renewal reward process. Yao and Relescu [28] apply the uncertain renewal process to analyze an age replacement policy. In addition, Yao [29] studies the uncertain calculus of the uncertain renewal process by proposing the integration and differentiation about the renewal process. Yao and $\mathrm{Li}$ [30] regard off-times and on-times as uncertain variables and propose an uncertain alternating renewal process. Zhang et al. [31] also show a delayed renewal process for uncertain interarrival times, where the first interarrival time is completely different from other times. Recently, Liu [32] provides an uncertain insurance risk model by applying the renewal reward process and derives the ruin index. In the uncertain insurance risk model, Liu assumes that the premium is a real function proportional to time and the claim amount obeys an uncertain renewal reward process. Based on Liu's insurance risk process, Yao and Zhou [33] further investigate the uncertainty distribution of the ruin time. Yao and Qin [34] point out that the premiums follow the renewal reward process in actual applications rather than a real function, so they propose an uncertain insurance risk process in which both the premiums and claims follow the uncertain renewal reward process. Liu et al. [35] extend Liu's model and discuss an uncertain insurance risk model with a variational lower limit. Liu and Yang [36] establish an uncertain insurance risk process considering an insurance company having multiple claims with uncertainty theory.

In the above studies, the claim process is regarded as a renewal process or a renewal reward process. In fact, the moment of the accident and the moment of the payment are not simultaneous, and in many types of insurance, the time interval between a claim event and the determination of the payment for the claim can be very long [37]. So, the claim process should include two processes: the report process and the payment process. The report process refers to the insured formally notifying the insurance company about an event. The payment process refers to the process whereby the insurer reviews the claim and sees whether the event or situation falls within the risks covered by the policy. That is to say, the insurer will need to determine that the claim meets the terms and conditions of the insurance policy. Obviously, the two processes should follow different uncertainty distributions. Therefore, it is more reasonable to view the claim process as an uncertain alternating renewal reward process. At present, few studies have considered the claim process in an insurance risk model as an uncertain alternating renewal process.

Inspired by the ideas we have reviewed, this paper proposes an insurance risk model in which the claims follow an uncertain alternating renewal reward process, while the premiums follow an uncertain renewal reward process. The inverse uncertainty distribution of insurance risk process, ruin index, and ruin time are derived. We also compare our model with Yao and Qin's model through numerical examples and explain the significance of describing the claim process by uncertain alternating renewal reward process.

The rest of the paper is organized as follows. Section 2 presents an uncertain alternating renewal insurance risk model and gives the expressions of ruin index and ruin time. In Section 3, several examples are provided to clarify the modeling idea of the insurance risk model. Finally, conclusions will be listed at the end.

\section{An Uncertain Alternating Renewal Insurance Risk Model}

Next, we study an insurance risk process in an uncertain environment. The premium process and the claim process are regarded as an uncertain renewal reward process and an uncertain alternating renewal reward process, respectively. In the following discussions, we give an assumption that the new claim event will not occur during the period of reviewing the current claim. Let the premium process be an uncertain renewal reward process:

$$
R_{1 t}=\sum_{i=1}^{N_{1 t}} P_{i},
$$

where $P_{1}, P_{2}, \ldots$ are independent uncertain premium amounts, and

$$
N_{1 t}=\max _{n \geq 0}\left\{n \mid \xi_{11}+\xi_{12}+\cdots+\xi_{1 n} \leq t\right\}
$$

is an uncertain renewal process with independent uncertain interarrival times $\xi_{11}, \xi_{12}, \ldots$.

The claim process is an uncertain alternating renewal reward process:

$$
R_{2 t}=\sum_{i=1}^{N_{2 t}} C_{i}
$$

where $C_{1}, C_{2}, \ldots$ are independent uncertain claim amounts. Consider the claim process as an uncertain alternating renewal reward process, and the claim process can be described as the following process. The first event happens as well as the insured reports claim for an uncertain time $\xi_{21}$. After an uncertain time $\xi_{21}$, the insurer reviews your claim and provides a payout for an uncertain time $\eta_{21}$ and at an uncertain claim amount $C_{1}$. Next, the second event happens and the insured reports claim for an uncertain time $\xi_{22}$. After an uncertain time $\xi_{22}$, the insurer reviews your claim and provides a payout for an uncertain time $\eta_{22}$ and at an uncertain claim amount $C_{2}$. The process continues infinitely (see Figure 1). Then, let

$$
\begin{aligned}
N_{2 t} & =\max _{n \geq 0}\left\{n \mid\left(\xi_{21}+\eta_{21}\right)+\left(\xi_{22}+\eta_{22}\right)+\cdots+\left(\xi_{2 n}+\eta_{2 n}\right) \leq t\right\} \\
& =\sup _{n \geq 0}\left\{n \mid 0 \leq\left(\xi_{21}+\eta_{21}\right)+\left(\xi_{22}+\eta_{22}\right)+\cdots+\left(\xi_{2 n}+\eta_{2 n}\right)\right. \\
& \left.\leq t<\left(\xi_{21}+\eta_{21}\right)+\left(\xi_{22}+\eta_{22}\right)+\cdots+\left(\xi_{2 n}+\eta_{2 n}\right)+\xi_{2 n+1}\right\},
\end{aligned}
$$

be an uncertain alternating renewal process, where $\xi_{21}, \xi_{22}, \ldots$ and $\eta_{21}, \eta_{22}, \ldots$ are independent uncertain 


$\xi_{21}, \eta_{21}, \xi_{22}, \eta_{22}, \ldots \ldots, \xi_{2 n}, \eta_{2 n}$,

FIgURE 1: Alternating renewal process.

interarrival times. $S_{n}=\left(\xi_{21}+\eta_{21}\right)+\left(\xi_{22}+\eta_{22}\right)+\cdots+\left(\xi_{2 n}\right.$ $\left.+\eta_{2 n}\right)$ denotes the moment of the payment of the $n$th claim.

Let $a$ be the initial capital, then the capital of an insurance company at time $t$ is

$$
Z_{t}=a+R_{1 t}-R_{2 t} \text {. }
$$

The $Z_{t}$ is an insurance risk model with an uncertain alternating renewal reward process. Apparently, once $Z_{t}<0$, the insurance company faces the risk of ruin.

For an uncertain insurance risk model $Z_{t}$, in order to obtain some important theorems, the following notations will be used:

$\Phi$ : the uncertainty distribution of the premium amount $P_{1}$ $\Psi$ : the uncertainty distribution of the claim amount $C_{1}$ $\mu_{1}$ : the uncertainty distribution of the interarrival time $\xi_{11}$ $\mu_{2}$ : the uncertainty distribution of the interarrival time $\xi_{21}$ $\lambda$ : the uncertainty distribution of the interarrival time $\eta_{21}$

2.1. Ruin Index. The ruin index can be defined as the uncertain measure that the capital $Z_{t}<0$ at time $t$. This section derives the explicit forms of the ruin index. Firstly, we get the inverse uncertainty distribution of an uncertain insurance risk process.

Theorem 1. For an uncertain insurance risk process $Z_{t}=a+R_{1 t}-R_{2 t}$, the inverse uncertainty distribution of $Z_{t}$ can be derived as

$$
\Upsilon_{t}^{-1}(\alpha)=a+\frac{\Phi^{-1}(\alpha)}{\mu_{1}^{-1}(1-\alpha)} t-\frac{\Psi^{-1}(\alpha)}{\mu_{2}^{-1}(\alpha)+\lambda^{-1}(\alpha)} t .
$$

Proof. Note that, for any

$$
\gamma \in \bigcup_{i=1}^{\infty}\left\{\left(\xi_{1 i} \geq \mu_{1}^{-1}(1-\alpha)\right) \cap\left(P_{i} \leq \Phi^{-1}(\alpha)\right) \cap\left(\xi_{2 i}+\eta_{2 i} \leq \mu_{2}^{-1}(\alpha)+\lambda_{2}^{-1}(\alpha)\right) \cap\left(C_{i} \geq \Psi^{-1}(1-\alpha)\right)\right\},
$$

we have

$$
\begin{aligned}
Z_{t}(\gamma)= & a+\sum_{i=1}^{N_{1 t}(\gamma)} P_{i}-\sum_{i=1}^{N_{2 t}(\gamma)} C_{i} \\
\leq & a+\frac{\Phi^{-1}(\alpha)}{\mu_{1}^{-1}(1-\alpha)} t-\frac{\Psi^{-1}(\alpha)}{\mu_{2}^{-1}(\alpha)+\lambda^{-1}(\alpha)} t, \\
& \left\{Z_{t}(\gamma) \leq a+\frac{\Phi^{-1}(\alpha)}{\mu_{1}^{-1}(1-\alpha)} t-\frac{\Psi^{-1}(\alpha)}{\mu_{2}^{-1}(\alpha)+\lambda^{-1}(\alpha)} t\right\} \\
\supset & \bigcup_{i=1}^{\infty}\left\{\left(\xi_{1 i} \geq \mu_{1}^{-1}(1-\alpha)\right) \cap\left(P_{i} \leq \Phi^{-1}(\alpha)\right) \cap\left(\xi_{2 i}+\eta_{2 i} \leq \mu_{2}^{-1}(\alpha)+\lambda_{2}^{-1}(\alpha)\right) \cap\left(C_{i} \geq \Psi^{-1}(1-\alpha)\right)\right\} .
\end{aligned}
$$

Since the uncertain variables are independent and according to the property of uncertain measure, we can obtain

$$
\begin{aligned}
M & \left\{\bigcap_{i=1}^{\infty}\left(\xi_{1 i} \geq \mu_{1}^{-1}(1-\alpha)\right) \cap\left(P_{i} \leq \Phi^{-1}(\alpha)\right) \cap\left(\xi_{2 i}+\eta_{2 i} \leq \mu_{2}^{-1}(\alpha)+\lambda_{2}^{-1}(\alpha)\right) \cap\left(C_{i} \geq \Psi^{-1}(1-\alpha)\right)\right\} \\
& =\bigwedge_{i=1}^{\infty} M\left\{\xi_{1 i} \geq \mu_{1}^{-1}(1-\alpha)\right\} \wedge M\left\{P_{i} \leq \Phi^{-1}(\alpha)\right\} \wedge M\left\{\xi_{2 i}+\eta_{2 i} \leq \mu_{2}^{-1}(\alpha)+\lambda_{2}^{-1}(\alpha)\right\} \wedge M\left\{C_{i} \geq \Psi^{-1}(1-\alpha)\right\} \\
& =\bigwedge_{i=1}^{\infty} \alpha \wedge \alpha \wedge \alpha \wedge \alpha \\
& =\alpha .
\end{aligned}
$$


Furthermore, it follows from the monotonicity of unIn addition, since for any certain measure that

$$
M\left\{Z_{t}(\gamma) \leq a+\frac{\Phi^{-1}(\alpha)}{\mu_{1}^{-1}(1-\alpha)} t-\frac{\Psi^{-1}(\alpha)}{\mu_{2}^{-1}(\alpha)+\lambda^{-1}(\alpha)} t\right\} \geq \alpha .
$$

$$
\gamma \in \bigcap_{i=1}^{\infty}\left\{\left(\xi_{1 i}<\mu_{1}^{-1}(1-\alpha)\right) \cap\left(P_{i}>\Phi^{-1}(\alpha)\right) \cap\left(\xi_{2 i}+\eta_{2 i}>\mu_{2}^{-1}(\alpha)+\lambda_{2}^{-1}(\alpha)\right) \cap\left(C_{i}<\Psi^{-1}(1-\alpha)\right)\right\}
$$

we have

$$
\begin{aligned}
Z_{t}(\gamma)= & a+\sum_{i=1}^{N_{1 t}(\gamma)} P_{i}-\sum_{i=1}^{N_{2 t}(\gamma)} C_{i} \\
> & a+\frac{\Phi^{-1}(\alpha)}{\mu_{1}^{-1}(1-\alpha)} t-\frac{\Psi^{-1}(\alpha)}{\mu_{2}^{-1}(\alpha)+\lambda^{-1}(\alpha)} t, \\
& \left\{Z_{t}(\gamma)>a+\frac{\Phi^{-1}(\alpha)}{\mu_{1}^{-1}(1-\alpha)} t-\frac{\Psi^{-1}(\alpha)}{\mu_{2}^{-1}(\alpha)+\lambda^{-1}(\alpha)} t\right\} \\
\supset & \bigcap_{i=1}^{\infty}\left\{\left(\xi_{1 i}<\mu_{1}^{-1}(1-\alpha)\right) \cap\left(P_{i}>\Phi^{-1}(\alpha)\right) \cap\left(\xi_{2 i}+\eta_{2 i}>\mu_{2}^{-1}(\alpha)+\lambda_{2}^{-1}(\alpha)\right) \cap\left(C_{i}<\Psi^{-1}(1-\alpha)\right)\right\} .
\end{aligned}
$$

According to the independence of these uncertain variables again that

$$
\begin{aligned}
M & \left\{\bigcup_{i=1}^{\infty}\left(\xi_{1 i}<\mu_{1}^{-1}(1-\alpha)\right) \cap\left(P_{i}>\Phi^{-1}(\alpha)\right) \cap\left(\xi_{2 i}+\eta_{2 i}>\mu_{2}^{-1}(\alpha)+\lambda_{2}^{-1}(\alpha)\right) \cap\left(C_{i}<\Psi^{-1}(1-\alpha)\right)\right\} \\
& =\bigwedge_{i=1}^{\infty} M\left\{\xi_{1 i}<\mu_{1}^{-1}(1-\alpha)\right\} \wedge M\left\{P_{i}>\Phi^{-1}(\alpha)\right\} \wedge M\left\{\xi_{2 i}+\eta_{2 i}>\mu_{2}^{-1}(\alpha)+\lambda_{2}^{-1}(\alpha)\right\} \wedge M\left\{C_{i}<\Psi^{-1}(1-\alpha)\right\} \\
& =\bigwedge_{i=1}^{\infty}(1-\alpha) \wedge(1-\alpha) \wedge(1-\alpha) \wedge(1-\alpha) \\
& =1-\alpha,
\end{aligned}
$$

we get

$$
M\left\{Z_{t}(\gamma)>a+\frac{\Phi^{-1}(\alpha)}{\mu_{1}^{-1}(1-\alpha)} t-\frac{\Psi^{-1}(\alpha)}{\mu_{2}^{-1}(\alpha)+\lambda^{-1}(\alpha)} t\right\} \geq 1-\alpha,
$$

which is equivalent to the following form (see duality of uncertain measure):

$$
M\left\{Z_{t}(\gamma) \leq a+\frac{\Phi^{-1}(\alpha)}{\mu_{1}^{-1}(1-\alpha)} t-\frac{\Psi^{-1}(\alpha)}{\mu_{2}^{-1}(\alpha)+\lambda^{-1}(\alpha)} t\right\} \leq \alpha .
$$

Above all, $M\left\{Z_{t}(\gamma) \leq a+\left(\left(\Phi^{-1}(\alpha)\right) /\left(\mu_{1}^{-1}(1-\alpha)\right)\right) t-\right.$ $\left.\left(\left(\Psi^{-1}(\alpha)\right) /\left(\mu_{2}^{-1}(\alpha)+\lambda^{-1}(\alpha)\right)\right) t\right\}=\alpha$.

Theorem 1 is proved.

Theorem 2. Let $Z_{t}=a+R_{1 t}-R_{2 t}$, the ruin index can be expressed as

$$
\text { ruin }=\max _{m \geq 0, n \geq 1} \alpha_{m, n}
$$

where $m$ and $n$ are nonnegative integers and

$$
\alpha_{m, n}=\sup \left\{\alpha \in[0,1] \mid n \mu_{2}^{-1}(\alpha)+n \lambda^{-1}(\alpha)-(m+1) \mu_{1}^{-1}(1-\alpha)<0, a+m \Phi^{-1}(\alpha)-n \Psi^{-1}(1-\alpha)<0\right\}
$$


Mathematical Problems in Engineering

5

Proof. Obviously, the ruin risk can be calculated by

$$
\left\{\inf _{t \geq 0} Z_{t}<0\right\}=\bigcup_{m=0}^{\infty} \bigcup_{n=1}^{\infty}\left\{\left(\sum_{j=1}^{n}\left(\xi_{2 j}+\eta_{2 j}\right)<\sum_{i=1}^{m+1} \xi_{1 i}\right) \cap\left(a+\sum_{i=1}^{m} P_{i}-\sum_{j=1}^{n} C_{j}<0\right)\right\} .
$$

(1) For given nonnegative $m$ and $n$,

$$
\begin{aligned}
& \left\{\left(\sum_{j=1}^{n}\left(\xi_{2 j}+\eta_{2 j}\right)<\sum_{i=1}^{m+1} \xi_{1 i}\right) \cap\left(a+\sum_{i=1}^{m} P_{i}-\sum_{j=1}^{n} C_{j}<0\right)\right\} \\
& \supset \bigcap_{i=1}^{m+1}\left\{\xi_{1 i}>\mu_{1}^{-1}\left(1-\alpha_{m, n}\right)\right\} \cap \bigcap_{i=1}^{m}\left\{P_{i} \leq \Phi^{-1}\left(\alpha_{m, n}\right)\right\} \cap \bigcap_{j=1}^{n}\left\{\left(\xi_{2 j}+\eta_{2 j} \leq \mu_{2}^{-1}\left(\alpha_{m, n}\right)+\lambda^{-1}\left(\alpha_{m, n}\right)\right) \cap\left(C_{j}>\Psi^{-1}\left(\alpha_{m, n}\right)\right)\right\} .
\end{aligned}
$$

Then, we have

$$
\begin{aligned}
M & \left\{\left(\sum_{j=1}^{n}\left(\xi_{2 j}+\eta_{2 j}\right)<\sum_{i=1}^{m+1} \xi_{1 i}\right) \cap\left(a+\sum_{i=1}^{m} P_{i}-\sum_{j=1}^{n} C_{j}<0\right)\right\} \\
\geq & \bigcap_{i=1}^{m+1}\left\{\xi_{1 i}>\mu_{1}^{-1}\left(1-\alpha_{m, n}\right)\right\} \cap \bigcap_{i=1}^{m}\left\{P_{i} \leq \Phi^{-1}\left(\alpha_{m, n}\right)\right\} \cap \bigcap_{j=1}^{n}\left\{\left(\xi_{2 j}+\eta_{2 j} \leq \mu_{2}^{-1}\left(\alpha_{m, n}\right)+\lambda^{-1}\left(\alpha_{m, n}\right)\right) \cap\left(C_{j}>\Psi^{-1}\left(\alpha_{m, n}\right)\right)\right\} \\
& \geq \bigwedge_{i=1}^{m+1} M\left\{\xi_{1 i}>\mu_{1}^{-1}\left(1-\alpha_{m, n}\right)\right\} \wedge \bigwedge_{i=1}^{m} M\left\{P_{i} \leq \Phi^{-1}\left(\alpha_{m, n}\right)\right\} \wedge \bigwedge_{j=1}^{n} M\left\{\left(\xi_{2 j}+\eta_{2 j} \leq \mu_{2}^{-1}\left(\alpha_{m, n}\right)+\lambda^{-1}\left(\alpha_{m, n}\right)\right) \wedge\left(C_{j}>\Psi^{-1}\left(\alpha_{m, n}\right)\right)\right\} \\
& =\alpha_{m, n}, \\
M\left\{\inf _{t \geq 0} Z_{t}<0\right\}= & M\left\{\bigcup_{m=0}^{\infty} \bigcup_{n=1}^{\infty}\left(\sum_{j=1}^{n}\left(\xi_{2 j}+\eta_{2 j}\right)<\sum_{i=1}^{m+1} \xi_{1 i}\right) \cap\left(a+\sum_{i=1}^{m} P_{i}-\sum_{j=1}^{n} C_{j}<0\right)\right\} \\
\geq & \max _{m \geq 0, n \geq 1} M\left\{\left(\sum_{j=1}^{n}\left(\xi_{2 j}+\eta_{2 j}\right)<\sum_{i=1}^{m+1} \xi_{1 i}\right) \cap\left(a+\sum_{i=1}^{m} P_{i}-\sum_{j=1}^{n} C_{j}<0\right)\right\} \\
= & \max _{m \geq 0, n \geq 1} \alpha_{m, n} .
\end{aligned}
$$

(2) For given nonnegative $m$ and $n$,

$$
\begin{aligned}
& \left\{\left(\sum_{j=1}^{n}\left(\xi_{2 j}+\eta_{2 j}\right)<\sum_{i=1}^{m+1} \xi_{1 i}\right) \cap\left(a+\sum_{i=1}^{m} P_{i}-\sum_{j=1}^{n} C_{j}<0\right)\right\} \\
& \quad \subset \bigcup_{i=1}^{m+1}\left\{\xi_{1 i}>\mu_{1}^{-1}\left(1-\alpha_{m, n}\right)\right\} \cup \bigcup_{i=1}^{m}\left\{P_{i} \leq \Phi^{-1}\left(\alpha_{m, n}\right)\right\} \cup \bigcup_{j=1}^{n}\left\{\left(\xi_{2 j}+\eta_{2 j} \leq \mu_{2}^{-1}\left(\alpha_{m, n}\right)+\lambda^{-1}\left(\alpha_{m, n}\right)\right) \cup\left(C_{j}>\Psi^{-1}\left(\alpha_{m, n}\right)\right)\right\} .
\end{aligned}
$$


Then, we also have

$$
\begin{aligned}
& \bigcup_{m=0}^{\infty} \bigcup_{n=1}^{\infty}\left\{\left(\sum_{j=1}^{n}\left(\xi_{2 j}+\eta_{2 j}\right)<\sum_{i=1}^{m+1} \xi_{1 i}\right) \cap\left(a+\sum_{i=1}^{m} P_{i}-\sum_{j=1}^{n} C_{j}<0\right)\right\} \\
& \subset \bigcup_{i=1}^{\infty} \bigcup_{n=1}^{\infty} \bigcup_{m=i-1}^{\infty}\left\{\xi_{1 i}>\mu_{1}^{-1}\left(1-\alpha_{m, n}\right)\right\} \cup \bigcup_{i=1}^{\infty} \bigcup_{n=1}^{\infty} \bigcup_{m=i-1}^{\infty}\left\{P_{i} \leq \Phi^{-1}\left(\alpha_{m, n}\right)\right\} \\
& \cup \bigcup_{j=1}^{\infty} \bigcup_{m=0}^{\infty} \bigcup_{n=j}^{\infty}\left\{\left(\xi_{2 j}+\eta_{2 j} \leq \mu_{2}^{-1}\left(\alpha_{m, n}\right)+\lambda^{-1}\left(\alpha_{m, n}\right)\right) \cup\left(C_{j}>\Psi^{-1}\left(\alpha_{m, n}\right)\right)\right\} \\
& =\bigcup_{i=1}^{\infty}\left\{\xi_{1 i}>\bigwedge_{n=1}^{\infty} \bigwedge_{m=i-1}^{\infty} \mu_{1}^{-1}\left(1-\alpha_{m, n}\right)\right\} \cup \bigcup_{i=1}^{\infty}\left\{P_{i} \leq \bigvee_{n=1}^{\infty} \bigvee_{m=i-1}^{\infty} \Phi^{-1}\left(\alpha_{m, n}\right)\right\} \\
& \cup \bigcup_{j=1}^{\infty}\left\{\left(\xi_{2 j}+\eta_{2 j} \leq \bigvee_{m=0}^{\infty} \bigvee_{n=j}^{\infty}\left(\mu_{2}^{-1}\left(\alpha_{m, n}\right)+\lambda^{-1}\left(\alpha_{m, n}\right)\right)\right) \cup\left(C_{j}>\bigwedge_{m=0}^{\infty} \bigwedge_{n=j}^{\infty} \Psi^{-1}\left(\alpha_{m, n}\right)\right)\right\} .
\end{aligned}
$$

Similarly, it follows from definition of uncertain measure that

$$
\begin{aligned}
& M\left\{\inf _{t \geq 0} Z_{t}<0\right\} \leq \bigvee_{i=1}^{\infty} M\left\{\xi_{1 i}>\bigwedge_{n=1}^{\infty} \bigwedge_{m=i-1}^{\infty} \mu_{1}^{-1}\left(1-\alpha_{m, n}\right)\right\} \vee \bigvee_{i=1}^{\infty} M\left\{P_{i} \leq \bigvee_{n=1}^{\infty} \stackrel{\vee}{m=i-1}^{\infty} \Phi^{-1}\left(\alpha_{m, n}\right)\right\} \\
& \vee \bigvee_{j=1}^{\infty} M\left\{\xi_{2 j}+\eta_{2 j} \leq \bigvee_{m=0}^{\infty} \bigvee_{n=j}^{\infty}\left(\mu_{2}^{-1}\left(\alpha_{m, n}\right)+\lambda^{-1}\left(\alpha_{m, n}\right)\right)\right\} \vee \bigvee_{j=1}^{\infty} M\left\{C_{j}>\bigwedge_{m=0}^{\infty} \bigwedge_{n=j}^{\infty} \Psi^{-1}\left(\alpha_{m, n}\right)\right\} \\
& =\max _{m, n \geq 0} \alpha_{m, n} .
\end{aligned}
$$

From (1) and (2), we can draw

$$
M\left\{\inf _{t \geq 0} Z_{t}<0\right\}=\max _{m, n \geq 0} \alpha_{m, n}
$$

Theorem 2 is proved.

Theorem 3. Let $Z_{t}=a+R_{1 t}-R_{2 t}$ be an uncertain alternating renewal insurance risk process. Then, the ruin index can be calculated by the following form:

$$
\begin{aligned}
\text { ruin } & =\max _{m \geq 0, n \geq 1} \sup _{x, y \geq 0}\left(1-\mu_{1}\left(\frac{x}{m+1}\right)\right) \wedge \mu_{2}\left(\frac{x}{2 n}\right) \wedge \lambda\left(\frac{x}{2 n}\right) \\
& \wedge \Phi\left(\frac{y}{m}\right) \wedge\left(1-\Psi\left(\frac{a+y}{n}\right)\right) .
\end{aligned}
$$

Proof. It is obvious that

$$
\begin{aligned}
& M\left\{\left(\sum_{j=1}^{n}\left(\xi_{2 j}+\eta_{2 j}\right)<\sum_{i=1}^{m+1} \xi_{1 i}\right) \cap\left(a+\sum_{i=1}^{m} P_{i}-\sum_{j=1}^{n} C_{j}<0\right)\right\} \\
& M\left\{\sum_{j=1}^{n}\left(\xi_{2 j}+\eta_{2 j}\right)<\sum_{i=1}^{m+1} \xi_{1 i}\right\} \wedge M\left\{a+\sum_{i=1}^{m} P_{i}-\sum_{j=1}^{n} C_{j}<0\right\} .
\end{aligned}
$$

Because we assume the uncertain interarrival times are independent, we have

$$
\begin{aligned}
& M\left\{\sum_{j=1}^{n}\left(\xi_{2 j}+\eta_{2 j}\right)<\sum_{i=1}^{m+1} \xi_{1 i}\right\} \\
& =M\left\{\sum_{j=1}^{n}\left(\xi_{2 j}+\eta_{2 j}\right)-\sum_{i=1}^{m+1} \xi_{1 i}<0\right\} \\
& =\sup _{x \geq 0}\left(1-\mu_{1}\left(\frac{x}{m+1}\right)\right) \wedge \mu_{2}\left(\frac{x}{2 n}\right) \wedge\left(\frac{x}{2 n}\right), \\
& M\left\{a+\sum_{i=1}^{m} P_{i}-\sum_{j=1}^{n} C_{j}<0\right\} \\
& \quad=\sup _{y \geq 0} \Phi\left(\frac{y}{m}\right) \wedge\left(1-\Psi\left(\frac{a+y}{n}\right)\right) .
\end{aligned}
$$

Hence,

$$
\operatorname{ruin}=M\left\{\inf _{t \geq 0} Z_{t}<0\right\}=\max _{m \geq 0, n \geq 1} \sup _{x, y \geq 0}\left(1-\mu_{1}\left(\frac{x}{m+1}\right)\right)
$$

$$
\wedge \mu_{2}\left(\frac{x}{2 n}\right) \wedge \lambda\left(\frac{x}{2 n}\right) \wedge \Phi\left(\frac{y}{m}\right) \wedge\left(1-\Psi\left(\frac{a+y}{n}\right)\right) .
$$

Theorem 3 is proved.

When uncertain variables $P_{1}, \xi_{11}, C_{1}, \xi_{21}$, and $\eta_{22}$ have determinate uncertainty distributions and corresponding inverse uncertainty distributions exist, we can calculate the 
crisp expressions of ruin index through Theorem 3 and Theorem 2, respectively.

2.2. Ruin Time. In addition to the ruin index, ruin time can also be used to measure the risk of an insurance company. Next, the definition of ruin time will be given, and the uncertainty distribution of ruin time can be derived.

Let $Z_{t}=a+\sum_{i=1}^{N_{1 t}} P_{i}-\sum_{j=1}^{N_{2 t}} C_{j}$ be the uncertain alternating renewal insurance risk process of an insurance company. Then, the ruin time of the insurance company can be defined as

$$
\tau=\inf \left\{t \geq 0 \mid Z_{t}<0\right\}
$$

It is easy to know that $\tau=+\infty$ means that the insurance company will not ruin. Therefore, for any $t \geq 0$, the ruin index also can be expressed in the following form:

$$
M\{\tau<+\infty\}=\lim _{t \longrightarrow \infty} M\{\tau \leq t\},
$$

where $M\{\tau \leq t\}$ denotes the uncertainty distribution of the ruin time.

We assume that the $n$th claim occurs at the instant $S_{n}=$ $\sum_{i=1}^{n}\left(\xi_{2 i}+\eta_{2 i}\right)$ and $N_{1 t}=m$ at this time. Thus, the capital of the insurance company at the $n$th claim is

$$
Y_{m, n}=a+\sum_{i=1}^{m} P_{i}-\sum_{j=1}^{n} C_{j}
$$

Then, we have

$$
\{\tau \leq t\}=\left\{\inf _{0 \leq s \leq t} Z_{s}<0\right\}=\bigcup_{m=0}^{\infty} \bigcup_{n=1}^{\infty}\left\{\sum_{i=1}^{m} \xi_{1 i} \leq t, \sum_{j=1}^{n}\left(\xi_{2 j}+\eta_{2 j}\right) \leq t, Y_{m, n}<0\right\} .
$$

The uncertain event $\left\{\sum_{i=1}^{m} \xi_{1 i} \leq t, \sum_{j=1}^{n}\left(\xi_{2 j}+\eta_{2 j}\right) \leq t, Y_{m, n}\right.$ $<0\}$ means that the $n$th claim occurs before the instant $t$, and the capital of the insurance company is less than 0 at this time.

$$
M\left\{\sum_{i=1}^{m} \xi_{1 i} \leq t, \sum_{j=1}^{n}\left(\xi_{2 j}+\eta_{2 j}\right) \leq t, Y_{m, n}<0\right\}=\alpha_{m, n}(t)
$$

Theorem 4. Suppose that the inverse uncertainty distributions of all the uncertain variables exist in the insurance risk

where process. Then,

$$
\begin{aligned}
\alpha_{m, n}(t)= & \sup \left\{\alpha \in[0,1] \mid m \mu_{1}^{-1}(\alpha) \leq t\right\} \wedge \sup \left\{\alpha \in[0,1] \mid n \mu_{2}^{-1}(\alpha)+n \lambda^{-1}(\alpha) \leq t\right\} \\
& \wedge \sup \left\{\alpha \in[0,1] \mid a+m \Phi^{-1}(\alpha)-n \Psi^{-1}(1-\alpha) \leq 0\right\} .
\end{aligned}
$$

Proof. Since

$$
\begin{aligned}
M & \left\{\sum_{i=1}^{m} \xi_{1 i} \leq t, \sum_{j=1}^{n}\left(\xi_{2 j}+\eta_{2 j}\right) \leq t, Y_{m, n}<0\right\}, \\
M & \left\{\sum_{i=1}^{m} \xi_{1 i} \leq t, \sum_{j=1}^{n}\left(\xi_{2 j}+\eta_{2 j}\right) \leq t, a+\sum_{i=1}^{m} P_{i}-\sum_{j=1}^{n} C_{j}<0\right\}, \\
\alpha_{m, n}(t)= & \sup \left\{\alpha \in[0,1] \mid m \mu_{1}^{-1}(\alpha) \leq t\right\} \wedge \sup \left\{\alpha \in[0,1] \mid n \mu_{2}^{-1}(\alpha)+n \lambda^{-1}(\alpha) \leq t\right\} \\
& \wedge \sup \left\{\alpha \in[0,1] \mid a+m \Phi^{-1}(\alpha)-n \Psi^{-1}(1-\alpha) \leq 0\right\},
\end{aligned}
$$

then, we have

$$
\begin{aligned}
& \left\{\sum_{i=1}^{m} \xi_{1 i} \leq t, \sum_{j=1}^{n}\left(\xi_{2 j}+\eta_{2 j}\right) \leq t, Y_{m, n}<0\right\}, \\
& \supset \bigcap_{i=1}^{m}\left\{\xi_{1 i} \leq \mu_{1}^{-1}\left(\alpha_{m, n}(t)\right)\right\} \bigcap \bigcap_{j=1}^{n}\left\{\left(\xi_{2 j}+\eta_{2 j}\right) \leq \mu_{2}^{-1}\left(\alpha_{m, n}(t)\right)+\lambda^{-1}\left(\alpha_{m, n}(t)\right)\right\} \\
& \bigcap \bigcap_{i=1}^{m}\left\{P_{i} \leq \Phi^{-1}\left(\alpha_{m, n}(t)\right)\right\} \bigcap \bigcap_{j=1}^{n}\left\{C_{j} \geq \Psi^{-1}\left(1-\alpha_{m, n}(t)\right)\right\} .
\end{aligned}
$$


According to the monotonicity of uncertain measure, it is obtained that

$$
\begin{aligned}
& M\left\{\sum_{i=1}^{m} \xi_{1 i} \leq t, \sum_{j=1}^{n}\left(\xi_{2 j}+\eta_{2 j}\right) \leq t, a+\sum_{i=1}^{m} P_{i}-\sum_{j=1}^{n} C_{j}<0\right\} \\
& \geq \bigwedge_{i=1}^{m} M\left\{\xi_{1 i} \leq \mu_{1}^{-1}\left(\alpha_{m, n}(t)\right)\right\} \wedge \bigwedge_{j=1}^{n} M\left\{\left(\xi_{2 j}+\eta_{2 j}\right) \leq \mu_{2}^{-1}\left(\alpha_{m, n}(t)\right)+\lambda^{-1}\left(\alpha_{m, n}(t)\right)\right\} \\
& \wedge \bigwedge_{i=1}^{m} M\left\{P_{i} \leq \Phi^{-1}\left(\alpha_{m, n}(t)\right)\right\} \wedge \bigwedge_{j=1}^{n}\left\{C_{j} \geq \Psi^{-1}\left(1-\alpha_{m, n}(t)\right)\right\} \\
& =\alpha_{m, n}(t) .
\end{aligned}
$$

Additionally, we have

$$
\begin{aligned}
& \left\{\sum_{i=1}^{m} \xi_{1 i} \leq t, \sum_{j=1}^{n}\left(\xi_{2 j}+\eta_{2 j}\right) \leq t, Y_{m, n}<0\right\} \\
& \quad \subset \bigcup_{i=1}^{m}\left\{\xi_{1 i} \leq \mu_{1}^{-1}\left(\alpha_{m, n}(t)\right)\right\} \cup \bigcup_{j=1}^{n}\left\{\left(\xi_{2 j}+\eta_{2 j}\right) \leq \mu_{2}^{-1}\left(\alpha_{m, n}(t)\right)+\lambda^{-1}\left(\alpha_{m, n}(t)\right)\right\} \\
& \quad \bigcup_{i=1}^{m}\left\{P_{i} \leq \Phi^{-1}\left(\alpha_{m, n}(t)\right)\right\} \cup \bigcup_{j=1}^{n}\left\{C_{j} \geq \Psi^{-1}\left(1-\alpha_{m, n}(t)\right)\right\} .
\end{aligned}
$$

Similarly, we can obtain

$$
\begin{aligned}
& M\left\{\sum_{i=1}^{m} \xi_{1 i} \leq t, \sum_{j=1}^{n}\left(\xi_{2 j}+\eta_{2 j}\right) \leq t, a+\sum_{i=1}^{m} P_{i}-\sum_{j=1}^{n} C_{j}<0\right\} \\
& \quad \leq \bigvee_{i=1}^{m} M\left\{\xi_{1 i} \leq \mu_{1}^{-1}\left(\alpha_{m, n}(t)\right)\right\} \vee \bigvee_{j=1}^{n} M\left\{\left(\xi_{2 j}+\eta_{2 j}\right) \leq \mu_{2}^{-1}\left(\alpha_{m, n}(t)\right)+\lambda^{-1}\left(\alpha_{m, n}(t)\right)\right\} \\
& \quad \vee \bigvee_{i=1}^{m} M\left\{P_{i} \leq \Phi^{-1}\left(\alpha_{m, n}(t)\right)\right\} \vee \bigvee_{j=1}^{n}\left\{C_{j} \geq \Psi^{-1}\left(1-\alpha_{m, n}(t)\right)\right\} \\
& \quad=\alpha_{m, n}(t) .
\end{aligned}
$$

Above all, we get

$$
M\left\{\sum_{i=1}^{m} \xi_{1 i} \leq t, \sum_{j=1}^{n}\left(\xi_{2 j}+\eta_{2 j}\right) \leq t, Y_{m, n}<0\right\}=\alpha_{m, n}(t) .
$$

Theorem 4 is proved.
Theorem 5. Suppose that the inverse uncertainty distributions of all the uncertain variables exist in the insurance risk process. Then, the ruin time $\tau$ has an uncertainty distribution:

$$
M\{\tau \leq t\}=\max _{m \geq 0, n \geq 1} \alpha_{m, n}(t),
$$

where

$$
\begin{aligned}
\alpha_{m, n}(t)= & \sup \left\{\alpha \in[0,1] \mid m \mu_{1}^{-1}(\alpha) \leq t\right\} \wedge \sup \left\{\alpha \in[0,1] \mid n \mu_{2}^{-1}(\alpha)+n \lambda^{-1}(\alpha) \leq t\right\} \\
& \left.\wedge \sup \{\alpha \in[0,]] \mid a+m \Phi^{-1}(\alpha)-n \Psi^{-1}(1-\alpha) \leq 0\right\} .
\end{aligned}
$$


Proof. Since

$$
\{\tau \leq t\}=\bigcup_{m=0}^{\infty} \bigcup_{n=1}^{\infty}\left\{\sum_{i=1}^{m} \xi_{1 i} \leq t, \sum_{j=1}^{n}\left(\xi_{2 j}+\eta_{2 j}\right) \leq t, a+\sum_{i=1}^{m} P_{i}-\sum_{j=1}^{n} C_{j}<0\right\},
$$

then, we have

$$
\begin{aligned}
& \bigcup_{m=0}^{\infty} \bigcup_{n=1}^{\infty}\left\{\sum_{i=1}^{m} \xi_{1 i} \leq t, \sum_{j=1}^{n}\left(\xi_{2 j}+\eta_{2 j}\right) \leq t, a+\sum_{i=1}^{m} P_{i}-\sum_{j=1}^{n} C_{j}<0\right\} \\
& \supset \bigcup_{m=0}^{\infty} \bigcup_{n=1}^{\infty} \bigcap_{i=1}^{m}\left\{\xi_{1 i} \leq \mu_{1}^{-1}\left(\alpha_{m, n}(t)\right)\right\} \cap \bigcup_{m=0}^{\infty} \bigcup_{n=1}^{\infty} \bigcap_{j=1}^{n}\left\{\left(\xi_{2 j}+\eta_{2 j}\right) \leq \mu_{2}^{-1}\left(\alpha_{m, n}(t)\right)+\lambda^{-1}\left(\alpha_{m, n}(t)\right)\right\} \\
& \bigcap \bigcup_{m=0}^{\infty} \bigcup_{n=1}^{\infty} \bigcap_{i=1}^{m}\left\{P_{i} \leq \Phi^{-1}\left(\alpha_{m, n}(t)\right)\right\} \cap \bigcup_{m=0}^{\infty} \bigcup_{n=1}^{\infty} \bigcap_{j=1}^{n}\left\{C_{j} \geq \Psi^{-1}\left(1-\alpha_{m, n}(t)\right)\right\} .
\end{aligned}
$$

According to the monotonicity of uncertain measure, it is obtained

$$
\begin{aligned}
& M\{\tau \leq t\} \\
& =M\left\{\bigcup_{m=0}^{\infty} \bigcup_{n=1}^{\infty}\left(\sum_{i=1}^{m} \xi_{1 i} \leq t, \sum_{j=1}^{n}\left(\xi_{2 j}+\eta_{2 j}\right) \leq t, a+\sum_{i=1}^{m} P_{i}-\sum_{j=1}^{n} C_{j}<0\right)\right\} \\
& \geq M\left\{\bigcup_{m=0}^{\infty} \bigcup_{n=1}^{\infty} \bigcap_{i=1}^{m}\left(\xi_{1 i} \leq \mu_{1}^{-1}\left(\alpha_{m, n}(t)\right)\right)\right\} \wedge M\left\{\bigcup_{m=0}^{\infty} \bigcup_{n=1}^{\infty} \bigcap_{j=1}^{n}\left\{\left(\left(\xi_{2 j}+\eta_{2 j}\right) \leq \mu_{2}^{-1}\left(\alpha_{m, n}(t)\right)+\lambda^{-1}\left(\alpha_{m, n}(t)\right)\right)\right\}\right\} \\
& \wedge M\left\{\bigcup_{m=0}^{\infty} \bigcup_{n=1}^{\infty} \bigcap_{i=1}^{m}\left\{\left(P_{i} \leq \Phi^{-1}\left(\alpha_{m, n}(t)\right)\right)\right\}\right\} \wedge M\left\{\bigcup_{m=0}^{\infty} \bigcup_{n=1}^{\infty} \bigcap_{j=1}^{n}\left(C_{j} \geq \Psi^{-1}\left(1-\alpha_{m, n}(t)\right)\right)\right\} \\
& =\bigvee_{m=0}^{\infty} \bigvee_{n=1}^{\infty} \wedge_{i=1}^{m} M\left\{\xi_{1 i} \leq \mu_{1}^{-1}\left(\alpha_{m, n}(t)\right)\right\} \wedge \underset{m=0}{\vee} \bigvee_{n=1}^{\infty} \bigwedge_{j=1}^{n} M\left\{\left(\xi_{2 j}+\eta_{2 j}\right) \leq \mu_{2}^{-1}\left(\alpha_{m, n}(t)\right)+\lambda^{-1}\left(\alpha_{m, n}(t)\right)\right\} \\
& \wedge \bigvee_{m=0}^{\infty} \bigvee_{n=1}^{\infty} \bigwedge_{i=1}^{m} M\left\{P_{i} \leq \Phi^{-1}\left(\alpha_{m, n}(t)\right)\right\} \wedge \bigvee_{m=0}^{\infty} \bigvee_{n=1}^{\infty} \bigwedge_{j=1}^{n} M\left\{C_{j} \geq \Psi^{-1}\left(1-\alpha_{m, n}(t)\right)\right\} \\
& =\bigvee_{m=0}^{\infty} \underset{n=1}{\vee} \alpha_{m, n}(t) \\
& =\max _{m \geq 0, n \geq 1} \alpha_{m, n}(t) \text {. }
\end{aligned}
$$

Also, since

$$
\begin{aligned}
& \bigcup_{m=0}^{\infty} \bigcup_{n=1}^{\infty}\left\{\sum_{i=1}^{m} \xi_{1 i} \leq t, \sum_{j=1}^{n}\left(\xi_{2 j}+\eta_{2 j}\right) \leq t, a+\sum_{i=1}^{m} P_{i}-\sum_{j=1}^{n} C_{j}<0\right\} \\
& \subset \bigcup_{m=0}^{\infty} \bigcup_{n=1}^{\infty} \bigcup_{i=1}^{m}\left\{\xi_{1 i} \leq \mu_{1}^{-1}\left(\alpha_{m, n}(t)\right)\right\} \bigcup \bigcup_{m=0}^{\infty} \bigcup_{n=1}^{\infty} \bigcup_{j=1}^{n}\left\{\left(\xi_{2 j}+\eta_{2 j}\right) \leq \mu_{2}^{-1}\left(\alpha_{m, n}(t)\right)+\lambda^{-1}\left(\alpha_{m, n}(t)\right)\right\} \\
& \cup \bigcup_{m=0}^{\infty} \bigcup_{n=1}^{\infty} \bigcup_{i=1}^{m}\left\{P_{i} \leq \Phi^{-1}\left(\alpha_{m, n}(t)\right)\right\} \bigcup \bigcup_{m=0}^{\infty} \bigcup_{n=1}^{\infty} \bigcup_{j=1}^{n}\left\{C_{j} \geq \Psi^{-1}\left(1-\alpha_{m, n}(t)\right)\right\} \\
& =\bigcup_{i=1}^{\infty} \bigcup_{m=i}^{\infty} \bigcup_{n=1}^{m}\left\{\xi_{1 i} \leq \mu_{1}^{-1}\left(\alpha_{m, n}(t)\right)\right\} \bigcup \bigcup_{j=1}^{\infty} \bigcup_{n=j}^{\infty} \bigcup_{m=0}^{\infty}\left\{\left(\xi_{2 j}+\eta_{2 j}\right) \leq \mu_{2}^{-1}\left(\alpha_{m, n}(t)\right)+\lambda^{-1}\left(\alpha_{m, n}(t)\right)\right\} \\
& \cup \bigcup_{i=1}^{\infty} \bigcup_{m=i}^{\infty} \bigcup_{n=1}^{m}\left\{P_{i} \leq \Phi^{-1}\left(\alpha_{m, n}(t)\right)\right\} \bigcup \bigcup_{j=1}^{\infty} \bigcup_{n=j}^{\infty} \bigcup_{m=0}^{\infty}\left\{C_{j} \geq \Psi^{-1}\left(1-\alpha_{m, n}(t)\right)\right\} \\
& =\bigcup_{i=1}^{\infty}\left\{\xi_{1 i} \leq \bigcup_{m=i}^{\infty} \bigcup_{n=1}^{\infty} \mu_{1}^{-1}\left(\alpha_{m, n}(t)\right)\right\} \bigcup \bigcup_{j=1}^{\infty}\left\{\left(\xi_{2 j}+\eta_{2 j}\right) \leq \bigcup_{n=j}^{\infty} \bigcup_{m=0}^{\infty}\left(\mu_{2}^{-1}\left(\alpha_{m, n}(t)\right)\right)+\lambda^{-1}\left(\alpha_{m, n}(t)\right)\right\} \\
& \cup \bigcup_{i=1}^{\infty}\left\{P_{i} \leq \bigcup_{m=i}^{\infty} \bigvee_{n=1}^{\infty} \Phi^{-1}\left(\alpha_{m, n}(t)\right)\right\} \bigcup \bigcup_{j=1}^{\infty}\left\{C_{j} \geq \bigwedge_{n=j}^{\infty} \bigwedge_{m=0}^{\infty} \Psi^{-1}\left(1-\alpha_{m, n}(t)\right)\right\},
\end{aligned}
$$


then, we have

$$
\begin{aligned}
& M\{\tau \leq t\} \\
& =M\left\{\bigcup_{m=0}^{\infty} \bigcup_{n=1}^{\infty}\left(\sum_{i=1}^{m} \xi_{1 i} \leq t, \sum_{j=1}^{n}\left(\xi_{2 j}+\eta_{2 j}\right) \leq t, a+\sum_{i=1}^{m} P_{i}-\sum_{j=1}^{n} C_{j}<0\right)\right\} \\
& \leq M\left\{\bigcup_{i=1}^{\infty}\left(\xi_{1 i} \leq \bigvee_{m=i}^{\infty} \bigcup_{n=1}^{\infty} \mu_{1}^{-1}\left(\alpha_{m, n}(t)\right)\right)\right\} \cup M\left\{\bigcup_{j=1}^{\infty}\left(\left(\xi_{2 j}+\eta_{2 j}\right) \leq \bigvee_{n=j}^{\infty} \bigvee_{m=0}^{\infty}\left(\mu_{2}^{-1}\left(\alpha_{m, n}(t)\right)+\lambda^{-1}\left(\alpha_{m, n}(t)\right)\right)\right)\right\} \\
& \cup M\left\{\bigcup_{i=1}^{\infty}\left(P_{i} \leq \bigcup_{m=i}^{\infty} \bigvee_{n=1}^{\infty} \Phi^{-1}\left(\alpha_{m, n}(t)\right)\right)\right\} \cup M\left\{\bigcup_{j=1}^{\infty}\left(C_{j} \geq \underset{n=j}{\infty} \stackrel{\wedge}{m=0}^{\infty} \Psi^{-1}\left(1-\alpha_{m, n}(t)\right)\right)\right\} \\
& \left.=\bigvee_{i=1}^{\infty} M\left\{\xi_{1 i} \leq \underset{m=i}{\vee} \bigvee_{n=1}^{\infty} \mu_{1}^{-1}\left(\alpha_{m, n}(t)\right)\right\} \vee \underset{j=1}{\vee} M\left\{\left(\xi_{2 j}+\eta_{2 j}\right) \leq \underset{n=j}{\vee} \underset{m=0}{\vee}\left(\mu_{2}^{-1}\left(\alpha_{m, n}(t)\right)+\lambda^{-1}\left(\alpha_{m, n}(t)\right)\right)\right\}\right\} \\
& \vee \bigvee_{i=1}^{\infty} M\left\{P_{i} \leq \underset{m=i}{\vee} \bigvee_{n=1}^{\infty} \Phi^{-1}\left(\alpha_{m, n}(t)\right)\right\} \vee \underset{j=1}{\vee} M\left\{C_{j} \geq \underset{n=j}{\stackrel{1}{\wedge} \bigwedge_{m=0}^{\infty}} \Psi^{-1}\left(1-\alpha_{m, n}(t)\right)\right\} \\
& =\bigvee_{i=1}^{\infty} \bigvee_{m=i}^{\infty} \bigvee_{n=1}^{\infty} \alpha_{m, n}(t) \vee \bigvee_{j=1}^{\infty} \bigvee_{n=j}^{\infty} \bigvee_{m=0}^{\infty} \alpha_{m, n}(t) \\
& =\max _{m \geq 0, n \geq 1} \alpha_{m, n}(t) \text {. }
\end{aligned}
$$

Thus, we get $M\{\tau \leq t\}=\max _{m \geq 0, n \geq 1} \alpha_{m, n}(t)$.

Theorem 5 is proved.

\section{Numerical Examples}

We will illustrate the above conclusions through the following five numerical examples and present how to calculate the ruin index and ruin time. Firstly, we compare our model with Yao and Qin's model [34] in Example 1 and compute the ruin index by inverse uncertainty distributions. Example 2 presents how to calculate the expected claim rate and the report rate according to the properties of uncertain renewal reward process [27] and uncertain alternating renewal process [30]. Example 3 and Example 4 give the uncertain measure that an insurance company ruins before time $t$. We compute the uncertainty distribution of ruin time in Example 5.

Example 1. Assume that $\mu_{1}=N\left(e_{1}, \sigma_{1}\right), \mu_{2}=N\left(e_{2}, \sigma_{2}\right)$, $\lambda=N\left(e_{3}, \sigma_{3}\right), \quad \Phi=N\left(e_{1}^{\prime}, \sigma_{1}^{\prime}\right)$, and $\Psi=N\left(e_{2}^{\prime}, \sigma_{2}^{\prime}\right)$ follow normal uncertainty distributions. Then, we have

$$
\begin{aligned}
n \mu_{2}^{-1}(\alpha)+n \lambda^{-1}(\alpha)-(m+1) \mu_{1}^{-1}(1-\alpha) \\
=n\left(e_{2}+\frac{\sigma_{2} \sqrt{3}}{\pi} \ln \frac{\alpha}{1-\alpha}\right)+n\left(e_{3}+\frac{\sigma_{3} \sqrt{3}}{\pi} \ln \frac{\alpha}{1-\alpha}\right)-(m+1)\left(e_{1}+\frac{\sigma_{1} \sqrt{3}}{\pi} \ln \frac{\alpha}{1-\alpha}\right) \\
a+m \Phi^{-1}(\alpha)-n \Psi^{-1}(1-\alpha) \\
=a+m\left(e_{1}^{\prime}+\frac{\sigma_{1}^{\prime} \sqrt{3}}{\pi} \ln \frac{\alpha}{1-\alpha}\right)-n\left(e_{2}^{\prime}+\frac{\sigma_{2}^{\prime} \sqrt{3}}{\pi} \ln \frac{1-\alpha}{\alpha}\right) .
\end{aligned}
$$

According to Theorem 2, we can obtain that

$$
\begin{aligned}
\alpha_{m, n} & =\left(1+\exp \left(\frac{\pi}{\sqrt{3}} \cdot \frac{n\left(e_{2}+e_{3}\right)-(m+1) e_{1}}{n\left(\sigma_{2}+\sigma_{3}\right)+(m+1) \sigma_{1}}\right)\right)^{-1} \\
& \wedge\left(1+\exp \left(\frac{\pi}{\sqrt{3}} \cdot \frac{a+m e_{1}^{\prime}-n e_{2}^{\prime}}{m \sigma_{1}^{\prime}+n \sigma_{2}^{\prime}}\right)\right)^{-1} .
\end{aligned}
$$

Furthermore, the ruin index can be calculated as

$$
\begin{aligned}
\text { ruin } & =\max _{m \geq 0, n \geq 1}\left(1+\exp \left(\frac{\pi}{\sqrt{3}} \cdot \frac{n\left(e_{2}+e_{3}\right)-(m+1) e_{1}}{n\left(\sigma_{2}+\sigma_{3}\right)+(m+1) \sigma_{1}}\right)\right)^{-1} \\
& \wedge\left(1+\exp \left(\frac{\pi}{\sqrt{3}} \cdot \frac{a+m e_{1}^{\prime}-n e_{2}^{\prime}}{m \sigma_{1}^{\prime}+n \sigma_{2}^{\prime}}\right)\right)^{-1} .
\end{aligned}
$$

We set $e_{1}=e_{2}=e_{3}=1, \sigma_{1}=\sigma_{2}=\sigma_{3}=1, e_{1}^{\prime}=e_{2}^{\prime}=3$, $\sigma_{1}^{\prime}=\sigma_{2}^{\prime}=3, a=1000$, and $m=n=80$ and calculate the risk 
index of the insurance company to be ruin $=0.0223$. In addition, when the initial capital $a$ varies from 0 to 1200, we calculate the ruin index and present the results in Figure 2. The results show that the ruin index falls from 0.3556 to 0.0106 when the initial capital $a$ increases from 0 to 1200 .

Note that if $e_{3}=0$ and $\sigma_{3}=0$ in Example 1, the ruin index degenerates into the ruin index of the modified insurance risk model proposed by Yao and Qin [34], that is, ruin $=\max _{m \geq 0, n \geq 1}\left(1+\exp \left((\pi / \sqrt{3}) \cdot\left(\left(n e_{2}-(m+1) e_{1}\right) /(n\right.\right.\right.$ $\left.\left.\left.\left.\sigma_{2}+(m+1) \sigma_{1}\right)\right)\right)\right)^{-1} \wedge\left(1+\exp \left((\pi / \sqrt{3}) \cdot\left(\left(a+m e_{1}^{\prime}-n e_{2}^{\prime}\right) /\right.\right.\right.$ $\left.\left.\left.\left(m \sigma_{1}^{\prime}+n \sigma_{2}^{\prime}\right)\right)\right)\right)^{-1}$. When other parameters are fixed, $\left(1+\exp \left((\pi / \sqrt{3}) \cdot\left(a+m e_{1}^{\prime}-n e_{2}^{\prime} / m \sigma_{1}^{\prime}+n \sigma_{2}^{\prime}\right)\right)\right)^{-1}$ decreases as the initial capital $a$ increases. The ruin index is equal to $\max _{m \geq 0, n \geq 1}\left(1+\exp \left((\pi / \sqrt{3}) \cdot\left(a+m e_{1}^{\prime}-n e_{2}^{\prime} / m \sigma_{1}^{\prime}+n \sigma_{2}^{\prime}\right)\right)\right)^{-1}$ when the initial capital $a$ is large enough. We can analyze that, with the increase in initial capital $a$, the ruin index calculated by the uncertain alternating renewal insurance risk model will tend to be the same as that computed by the model proposed by Yao and Qin.

Example 2. Assume that $\mu_{1}=L(0,1), \quad \mu_{2}=L(1,4)$, $\lambda=L(2,3), \quad P_{1}=L(0,10)$, and $C_{1}=L(0,15)$ are linear uncertain variables. Thus, the expected claim rate is

$$
\begin{aligned}
\lim _{t \rightarrow \infty} \frac{E\left[N_{2 t}\right]}{t} & =E\left[\frac{1}{\xi_{21}+\eta_{21}}\right] \\
& =\int_{0}^{1} \frac{1}{\mu_{2}^{-1}(\alpha)+\lambda^{-1}(\alpha)} \mathrm{d} \alpha \\
& =0.212,
\end{aligned}
$$

and the report rate is

$$
\begin{aligned}
\lim _{t \rightarrow \infty} \frac{E\left[A_{t}\right]}{t} & =E\left[\frac{\xi_{21}}{\xi_{21}+\eta_{21}}\right] \\
& =\int_{0}^{1} \frac{\mu_{2}^{-1}(\alpha)}{\mu_{2}^{-1}(\alpha)+\lambda^{-1}(1-\alpha)} \mathrm{d} \alpha \\
& =0.486 .
\end{aligned}
$$

Example 3. Assume that $\mu_{1}=L(0,1), \quad \mu_{2}=L(1,4)$, $\lambda=L(2,3), P_{1}=L(0,10), C_{1}=L(0,15)$, the initial capital $a=1000$, and premium amount $m=80$. We calculate the company ruins with an uncertain measure 0.1 before the instant $t=300$ at the $n=80$ th claim. Figure 3 shows the uncertain measure that the insurance company ruins before time $t$.

Example 4. Assume that $\mu_{1}=L(0,1), \quad \mu_{2}=L(1,4)$, $\lambda=L(2,3), \quad P_{1}=L(0,10)$, and $C_{1}=L(0,15)$ are linear uncertain variables. According to Theorem 5 , if the initial capital $a$ is 1000, then we can calculate that $M\{\tau \leq 300\}=0.1524$; i.e., the insurance company ruins with an uncertain measure 0.1524 before the time $t=300$.

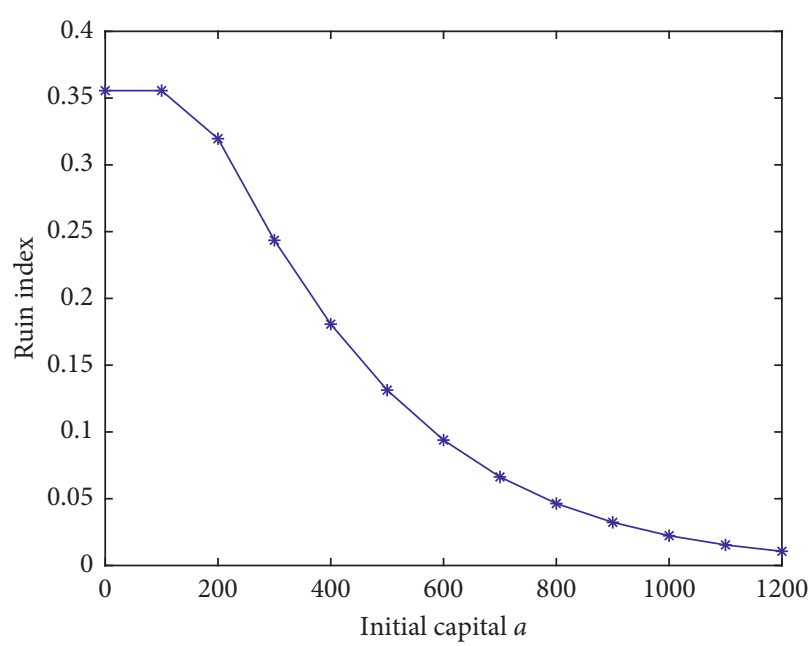

Figure 2: The ruin index with different initial capital $a$.

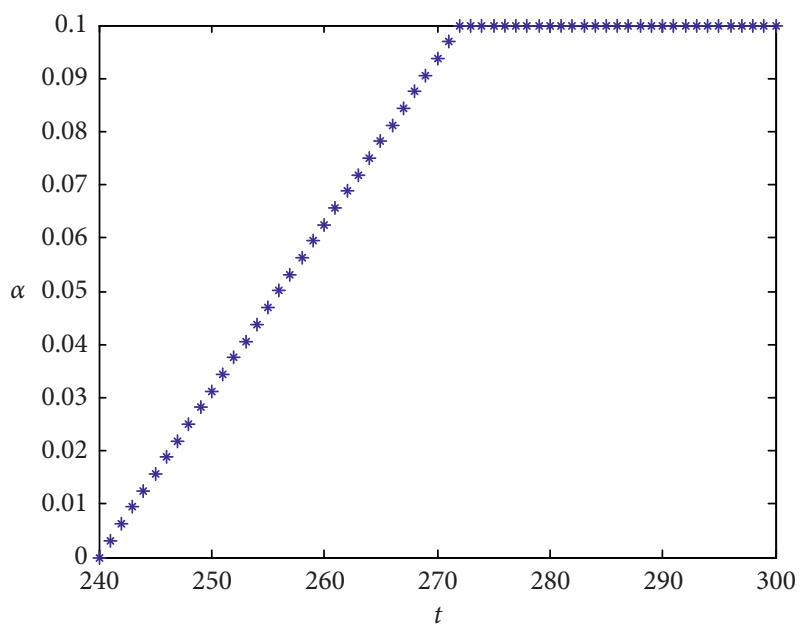

Figure 3: Risk measure of insurance company before the time $t$.

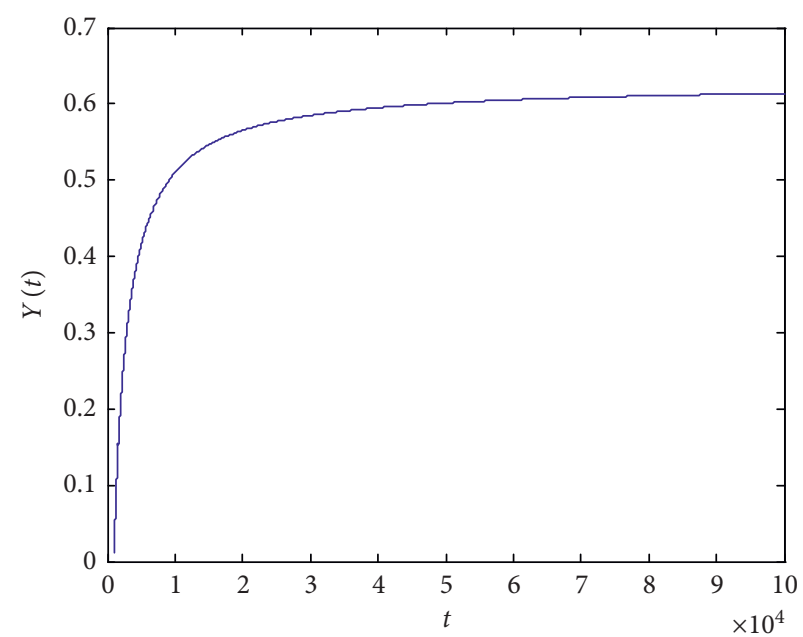

FIgURE 4: Uncertainty distribution of ruin time in Example 5. 
Example 5. Similar to Example 4, we assume that $\mu_{1}=L(0,1), \mu_{2}=L(1,4), \lambda=L(2,3), P_{1}=L(0,10)$, and $C_{1}=L(0,15)$ are linear uncertain variables. According to Theorem 5 , if the initial capital $a$ is 5000, the uncertainty distribution of the ruin time $\tau$ is shown in Figure 4 .

\section{Conclusions}

This paper extends an insurance risk model with an alternating renewal process in uncertain environment. We propose an uncertain insurance risk process in which the claim process is regarded as an uncertain alternating renewal reward process, and this process is more in line with the claim process in real life. Moreover, we provide the inverse uncertainty distribution of the uncertain insurance risk process and the ruin index. The explicit form of uncertainty distribution of ruin time is also derived. At last, several examples are provided to illustrate the proven results. In future research, some criteria such as inflation, dividend, and tax can be considered to further extend the uncertain insurance risk process.

\section{Data Availability}

The data presented in Examples 1-5 and Figures 2-4 in this paper, which are used to support the findings of this study, are included within the article.

\section{Conflicts of Interest}

The authors declare that they have no conflicts of interest.

\section{Acknowledgments}

This work was supported by the Beijing Natural Science Foundation (grant no. 9204024), National Natural Science Foundation of China (grant nos. 71873012 and 71571007), Humanities and Social Sciences Planning Fund of Ministry of Education (grant no. 17YJA790097), and Scientific Research Level Enhancement Funding Project of Beijing International Studies University.

\section{References}

[1] D. C. M. Dickson and C. Hipp, "Ruin probabilities for Erlang(2) risk processes," Insurance: Mathematics and Economics, vol. 22, no. 3, pp. 251-262, 1998.

[2] S. Li and J. Garrido, "On ruin for the Erlang(n) risk process," Insurance: Mathematics and Economics, vol. 34, no. 3, pp. 391-408, 2004.

[3] D. C. M. Dickson and C. Hipp, "On the time to ruin for Erlang(2) risk processes," Insurance: Mathematics and Economics, vol. 29, no. 3, pp. 333-344, 2001.

[4] Y. Chun, "Equivalence of axioms for bankruptcy problems," International Journal of Game Theory, vol. 28, no. 4, pp. 511-520, 1999.

[5] H. U. Gerber and E. S. W. Shiu, "On the time value of ruin," North American Actuarial Journal, vol. 2, no. 1, pp. 48-72, 1998.
[6] B. Sundt and J. L. Teugels, "Ruin estimates under interest force," Insurance: Mathematics and Economics, vol. 16, no. 1, pp. 7-22, 1995.

[7] J. Paulsen and H. K. Gjessing, "Optimal choice of dividend barriers for a risk process with stochastic return on investments," Insurance: Mathematics and Economics, vol. 20, no. 3, pp. 215-223, 1997.

[8] H. Albrecher and C. Hipp, "Lundberg's risk process with tax," Blätter der DGVFM, vol. 28, no. 1, pp. 13-28, 2007.

[9] W. Yu, P. Guo, Q. Wang et al., "On a periodic capital injection and barrier dividend strategy in the compound Poisson risk model," Mathematics, vol. 8, no. 4, p. 511, 2020.

[10] P. S. Griffin, "Sample path behavior of a Lévy insurance risk process approaching ruin, under the Cramér-Lundberg and convolution equivalent conditions," The Annals of Applied Probability, vol. 26, no. 1, pp. 360-401, 2016.

[11] E. Biffis and A. E. Kyprianou, "A note on scale functions and the time value of ruin for Lévy insurance risk processes," Insurance: Mathematics and Economics, vol. 46, no. 1, pp. 85-91, 2010.

[12] Z. Zhang, Y. Yong, and W. Yu, "Valuing equity-linked death benefits in general exponential Lévy models," Journal of Computational and Applied Mathematics, vol. 365, Article ID 112377, 2020.

[13] W. Yu, Y. Yong, G. Guan, Y. Huang, W. Su, and C. Cui, "Valuing guaranteed minimum death benefits by cosine series expansion," Mathematics, vol. 7, no. 9, p. 835, 2019.

[14] D. Kahneman and A. Tversky, "Prospect theory: an analysis of decision under risk," Econometrica, vol. 47, no. 2, pp. 263292, 1979.

[15] G. W. De Wit, "Underwriting and uncertainty," Insurance: Mathematics and Economics, vol. 1, no. 4, pp. 277-285, 1982.

[16] J. Lemaire, "Fuzzy insurance," ASTIN Bulletin, vol. 20, no. 1, pp. 33-55, 1990.

[17] J. D. Cummins and R. A. Derrig, "Fuzzy trends in propertyliability insurance claim costs," The Journal of Risk and Insurance, vol. 60, no. 3, pp. 429-465, 1993.

[18] R. A. Derrig and K. M. Ostaszewski, "Fuzzy techniques of pattern recognition in risk and claim classification," The Journal of Risk and Insurance, vol. 62, no. 3, pp. 447-482, 1995.

[19] W. Yu, F. Wang, Y. Huang, and H. Liu, "Social Optimal mean field control problem for population growth model," Asian Journal of Control, pp. 1-8, 2019.

[20] A. F. Shapiro, "Fuzzy logic in insurance," Insurance: Mathematics and Economics, vol. 35, no. 2, pp. 399-424, 2004.

[21] B. Li, W. Ni, and C. Constantinescu, "Risk models with premiums adjusted to claims number," Insurance: Mathematics and Economics, vol. 65, pp. 94-102, 2015.

[22] T. Huang, R. Zhao, and W. Tang, "Risk model with fuzzy random individual claim amount," European Journal of Operational Research, vol. 192, no. 3, pp. 879-890, 2009.

[23] A. F. Shapiro, "Modeling future lifetime as a fuzzy random variable," Insurance: Mathematics and Economics, vol. 53, no. 3, pp. 864-870, 2013.

[24] B. Liu, Uncertainty Theory, Springer, Berlin, Germany, 2007.

[25] S. Li, J. Peng, and B. Zhang, "The uncertain premium principle based on the distortion function," Insurance: Mathematics and Economics, vol. 53, no. 2, pp. 317-324, 2013.

[26] B. Liu, "Fuzzy process, hybrid process and uncertain process," Journal of Uncertain Systems, vol. 2, no. 1, pp. 3-16, 2008.

[27] B. Liu, Uncertainty Theory: A Branch of Mathematics for Modeling Human Uncertainty, Springer, Berlin, Germany, 2010. 
[28] K. Yao and D. A. Relescu, "Age replacement policy in uncertain environment," Iranian Journal of Fuzzy Systems, vol. 10, no. 2, pp. 29-39, 2013.

[29] K. Yao, "Uncertain calculus with renewal process," Fuzzy Optimization and Decision Making, vol. 11, no. 3, pp. 285-297, 2012.

[30] K. Yao and X. Li, "Uncertain alternating renewal process and its application," IEEE Transactions on Fuzzy Systems, vol. 20, no. 6, pp. 1154-1160, 2012.

[31] X. Zhang, Y. Ning, and G. Meng, "Delayed renewal process with uncertain interarrival times," Fuzzy Optimization and Decision Making, vol. 1, pp. 279-287, 2013.

[32] B. Liu, "Extreme value theorems of uncertain process with application to insurance risk model," Soft Computing, vol. 17, no. 4, pp. 549-556, 2013.

[33] K. Yao and J. Zhou, "Ruin time of uncertain insurance risk process," IEEE Transactions on Fuzzy Systems, vol. 26, no. 1, pp. 19-28, 2018.

[34] K. Yao and Z. Qin, "A modified insurance risk process with uncertainty," Insurance: Mathematics and Economics, vol. 62, pp. 227-233, 2015.

[35] Y. Liu, X. Zhang, and W. Ma, "A new uncertain insurance model with variational lower limit," Insurance: Mathematics and Economics, vol. 74, pp. 164-169, 2017.

[36] Z. Liu and Y. Yang, "Uncertain insurance risk process with multiple classes of claims," Applied Mathematical Modelling, vol. 83, pp. 660-673, 2020.

[37] H. Lopes, J. Barcellos, J. Kubrusly, and C. Fernandes, “A nonparametric method for incurred but not reported claim reserve estimation," International Journal for Uncertainty Quantification, vol. 2, no. 1, pp. 39-51, 2012. 